\title{
Assistive Technology for Intellectually Disabled and Physically Challenged People
}

\author{
Mr.Darshan.B \\ VIII sem BE, Dept of ECE \\ BNMIT, Banglore, India \\ Darshandak7347@gmail.com
}

\author{
Mr.Chethan Kumar.B.O \\ VIII sem BE, Dept of ECE \\ BNMIT, Banglore, India \\ bochethan@gmail.com
}

\author{
Dr.P.A.Vijaya \\ Professor and HOD, Dept of ECE \\ BNMIT, Banglore, India \\ pavmkv@gmail.com
}

\begin{abstract}
Assistive technology is an embedded system which receives inputs from the disabled and provides them the desired service. The system can either be a hard real time system such as health monitoring system or a soft real time system which takes up requests that can be serviced later. The device consists of a choice selection pad which receives the input from the user. The choice pad can be either wireless or wired. The pad can be mounted on a wall so that it can be used by many disabled people or a separate pad with a unique id can be provided to each user. The disabled person will enter his choice of service using the pad. The module on receiving the selection will display the service name on the LCD screen and a vocal message will be announced. The assistant who is serving the disabled persons will hear the vocal message and respond to it by providing the service requested. If in case the assistant is located in a remote place a message containing information about the service request will be sent to his cell phone using a GSM module attached to the assistive module. Since GSM modules are used each user will be having a unique SIM number for the module which he is using, this helps the assistant to know the exact module or the user from which the request is originated. To receive the requests from several modules we use a GSM module at the receiving end. The GSM module will be connected to the laptop which will serve as a server for the storage of the requests.
\end{abstract}

Keywords: ID, GSM, LCD and TMFT.

Abbreviations: GSM-global system for mobile communication, LCD-liquid crystal display, ID-intellectual disability and USB-universal serial bus.

\section{INTRODUCTION}

An embedded system is a computer system with a dedicated function within a larger mechanical or electrical system, often with real-time computing constraints. It is embedded as part of a complete device often including hardware and mechanical parts. Embedded systems control many devices in common use today. Modern embedded systems are often based on microcontrollers (i.e. CPUs with integrated memory or peripheral interfaces), but ordinary microprocessors (using external chips for memory and peripheral interface circuits) are also common, especially in more-complex systems.

Assistive technology is a technology designed to provide assistance to the consumer or the subscriber. The technology is widely used in the field of medicine, banking, and hotels and so on. Assistive technology can also be used to provide assistance to the intellectually disabled and physically challenged people. Intellectually disabled people are those people born with a limited skill set. They lack day-to-day skills but have an adaptive behaviour. They can do and learn new things within their skill set but they learn very slowly. Physically challenged people refer to blind people, lame people and dumb people. The country's disabled population has increased by $22.4 \%$ between 2001 and 2011 . The number of disabled, which was 2.19 crore in 2001, rose in 2011 to 2.68 crore- 1.5 crore males and 1.18 crore females. Assistive technology can be used to receive inputs from the disabled person and communicate them to a normal person so that the required services can be provided. The inputs can be in the form of signal via keypad, brain waves or gestures. 


\section{SYSTEM REQUIREMENTS}

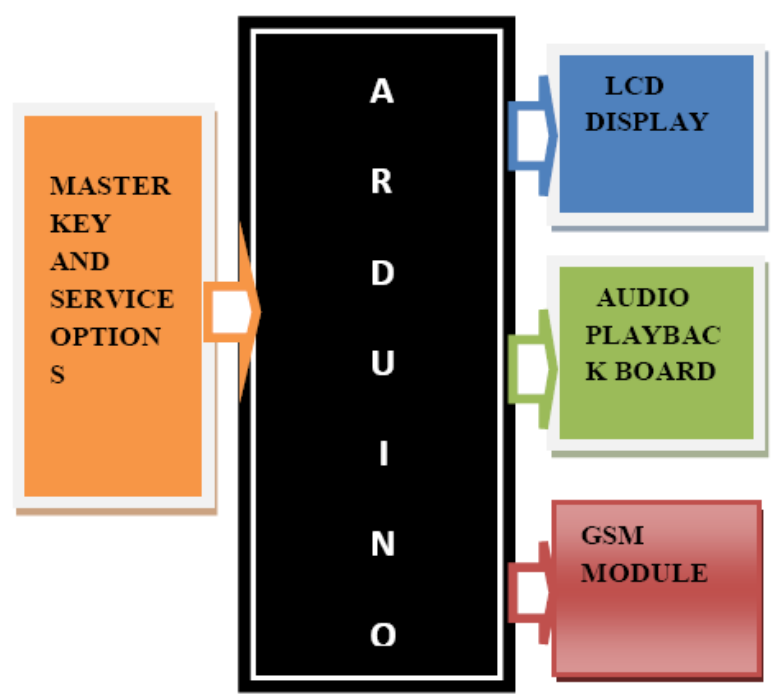

Fig2. System block diagram

The device receives the input from a $4 * 4$ hexagonal keypad using column scanning method. Once the input is received the message corresponding to the key pressed will be displayed on the $16 * 2 \mathrm{LCD}$ screen. The audio playback board now announces the same text message. To reach the assistant, who is in a remote place we use a GSM module with serial communication. The Arduino starts the serial communication at a baud rate 9600 bps using AT commands to control the GSM module. The assistant on hearing the message first confirms with the display and provides the service requested.

The hardware requirements are as follows

1. Choice selection pad

2. Arduino mega board

3. LCD display

4. GSM module

5. Audio playback board

The software requirements are

1. Arduino IDE

2. TMFT

\section{HaRdWare Design}

\subsection{Choice Selection Pad}

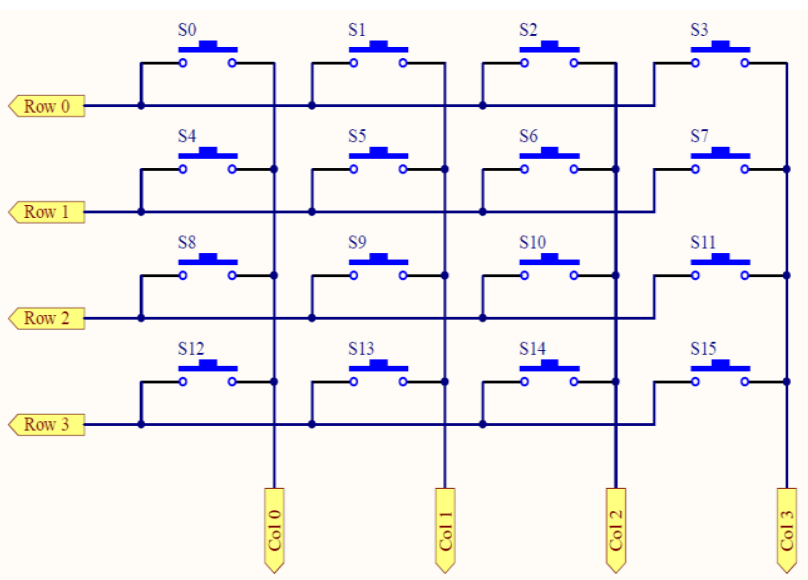

Fig3.1. Hexagonal keypad internal structure 
The choice selection pad is $4 * 4$ hexagonal keypad, which is connected to the port analog pins A0 to A7 of the arduino. The arduino uses the column scanning method to recognize the user input. 8 analog lines are connected to the row and column lines. The column scanning is used to reduce the number of pins required to recognize the input. For a 16 input choice selection pad, we need only 8 input lines. The keypad provides 16 inputs or choices, out of which one is identified as the master key. The priority is set in such a way that the master key as the highest priority. Any input provided without the press of master key will be recognized as an invalid input. Once the master key is pressed the arduino validates the key and polls the keypad for the actual choice. The rows and columns are numbered as R1, R2, R3, R4, C1, C2, C3 and C4 respectively.

\subsection{Arduino Mega 2560 R3 Development Board}

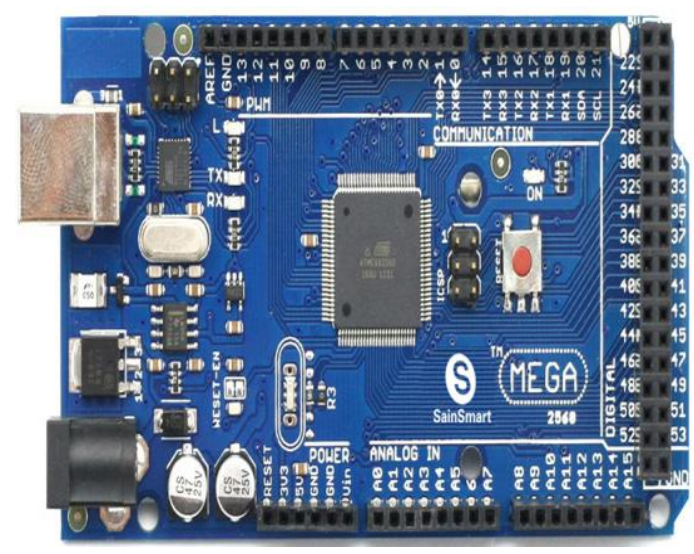

Fig3.2. Arduino mega development board

The arduino mega is the CPU of the assistive module. It performs the scanning of the input and initiates the appropriate modules required to provide the service. The Arduino Mega2560 can be powered via the USB connection or with an external power supply of 6 to 20 volts. The arduino mega board provides $256 \mathrm{~Kb}$ of flash memory. The advantage of using arduino mega board is that it supports the use of interrupts which can be used to convey the emergency conditions in hospitals. The arduino board on receiving the input will use a look up table to map every input to its respective output. The selection of channel for the voice message and the writing of data to the LCD are done by the arduino board. The arduino also interacts and writes data to the GSM module using the RS232 serial communication standards.

\subsection{LCD Module}
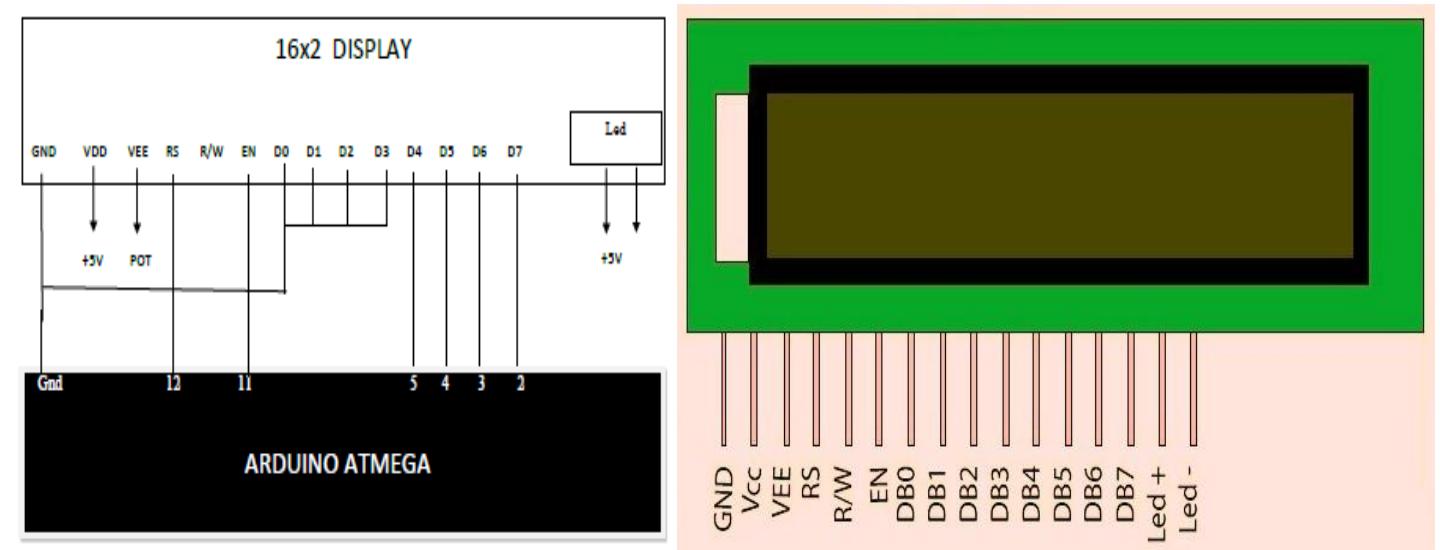

Fig3.3. LCD module interface

The liquid crystal display consists of 2 rows and 16 columns. The LCD is connected to the digital I/O of the arduino. The LCD used has 16 pins each of which are described as shown in fig 2.3.The VCC and GND are the power supply pins for the LCD. The VEE pin is used for the LCD contrast adjustment. This pin is usually connected to a potentiometer for achieving variable contrast. The R/W pin is to select the mode of operation of the LCD. We can either write to the LCD or read from it. The $\mathrm{R} / \mathrm{W}$ pin is connected to the ground, since we are only writing to the LCD. The RS pin is used for the selection of the register. The LCD is used in the 4 bit mode. The reason for using the LCD in the four 
bit mode is that it requires lesser (only 4 for data) number of pins to interface with the arduino board. The first four data pins are grounded and the remaining four are connected to the arduino I/O. In 4-bit mode the data is sent in nibbles, first we send the higher nibble and then the lower nibble. To interface the LCD with the arduino we use a header file <Liquidcrystal.h >. The header file provides the functions like lcd.println () which is used to write to the LCD, lcd.setcursor () to set the cursor position and lcd.clear () to clear the LCD screen.

\subsection{GSM Module - SIM 800A}

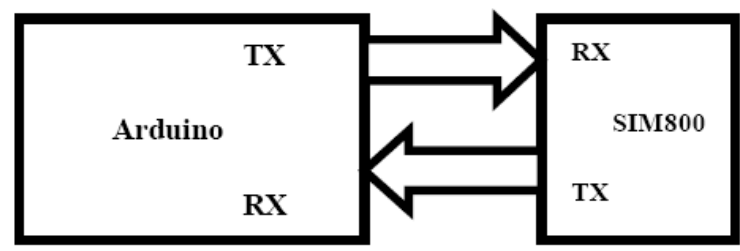

Fig3.4. GSM interface to arduino

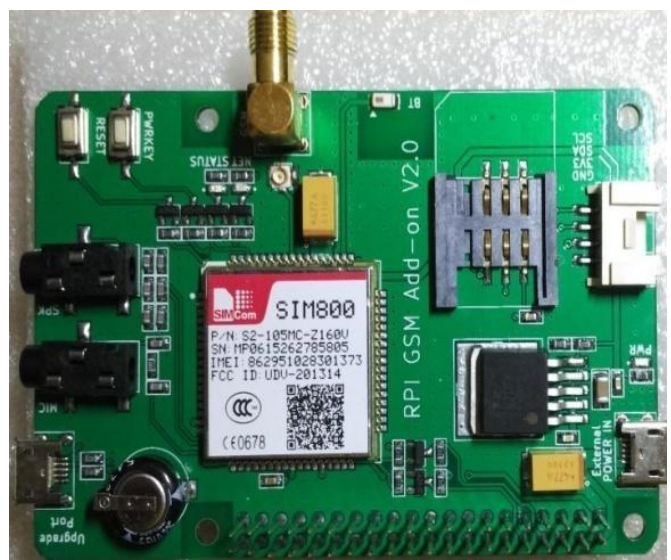

Fig3.5. SIM800 module

The arduino board communicates with the SIM 800 module using AT commands. AT stands for attention. At commands are used to gain the attention of the arduino for the exchange of information. . Many of the commands that are used to control wired dial-up modems, such as ATD(dial), Ata(answer), ATH(hook control), ATO(return to online data state), are also supported by GSM/GPRS modems and mobile phones. The assistive module uses the SIM800 module to send the messages hence the module has to operate in the text mode. $\mathrm{AT}+\mathrm{CMGF}=1$ instruction will configure the modem to text mode. Once the text mode is enabled we define the mobile number to which the SMS has to be sent using the AT+CMGS=I"+919449XXXXX|"\r". The message to be sent is written at a baud rate of 9600 .The messages sent by the assistive module will be received by the GSM module connected to a laptop at the control centre. The laptop is used as a server to know the requests made by the disabled people. The SIM card inserted in the GSM module will have storage of about 20 text messages. It is possible to read the messages using the command AT+CMGL="ALL". To read only a single request the $\mathrm{AT}+\mathrm{CMGR}=1$ instruction can be used.

\subsection{APR33A3 Audio Play Back Board}

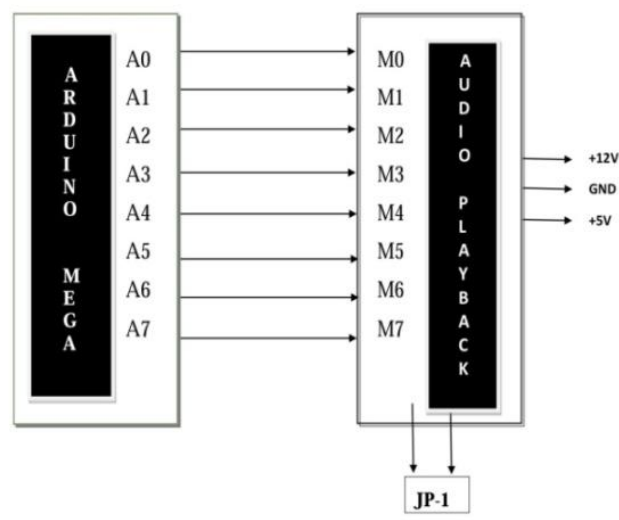

Fig3.6. Audio playback board interface 


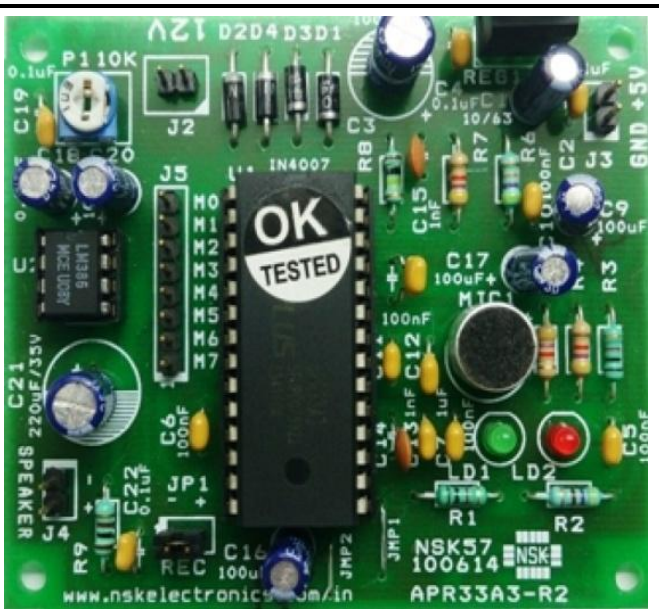

Fig3.7. Audio play board ARP33a-R2

The aPR33A series are powerful audio processor along with high performance audio analog-to-digital converters (ADCs) and digital-to-analog converters (DACs). The audio playback board is used to store the voice messages. The board provides a non-volatile flash memory and requires no battery backup. The messages are stored in the digital format as the board processes a 16 bit digital audio processor. The voice message from the user is converted to a digital format by a high quality analog to digital converter. The board as an integrated microphone which is used to record the voice messages. The messages should be recorded with a sampling rate of $4 \mathrm{KHz}$. The board as 8 voice channels and each channel has storage of 1.3 minutes. The audio play back board operates in two modes, namely the record mode and the playback mode.

\section{Operation}

\section{a. Record mode}

1. Provide $12 \mathrm{v}$ DC as supply to board. On board regulator converts it to $5 \mathrm{v}$ for the IC.

2. Put Jumper JP1 for RECORD mode.

3. While in record mode choose any pin from J5 (M0-M7) to select a channel to record the message. For e.g. to record message in channel M0 ,

4. Connect M0 to GND (you can use Jumper J3 GND pin).

5. Speak through the MIC .The LED LD2 will be ON indicating REC is ON. Once the duration is full (1.3 minutes) the LED goes OFF.

6. Now disconnect the GND Connection from M0 .If you remove this connection in between, the rest duration for that message slot is kept empty.

\section{b. Playback mode}

1. Connect the speaker to the board J4 Speaker section.

2. Remove jumper from JP1(REC)section

3. Now connect the MO (of J5) to GND.

4. Status LED (LD2) will be ON till the recorded sound play in the speaker.

\section{SofTWARE DESIGN}

The two software tools used in the design of the module are Arduino IDE and the TMFT. The opensource Arduino Software (IDE) makes it easy to write code and upload it to the board. It runs on Windows, Mac OS X, and Linux. The environment is written in Java and based on Processing and other open-source software.

Arduino programs can be written in any of the programming languages like c, c++, Java etc... The arduino program should always contain 2 block, namely the void setup ( ) and void loop ( ). The instructions which are to be executed once will be written inside the setup ( ) block and the instructions that are to be executed will be written inside the loop ( ) block. 
The Arduino communicates with the computer using serial communication hence it is required that during the download of the program into the arduino kit the GSM module should be detached. If the GSM is connected the downloading will be unsuccessful.

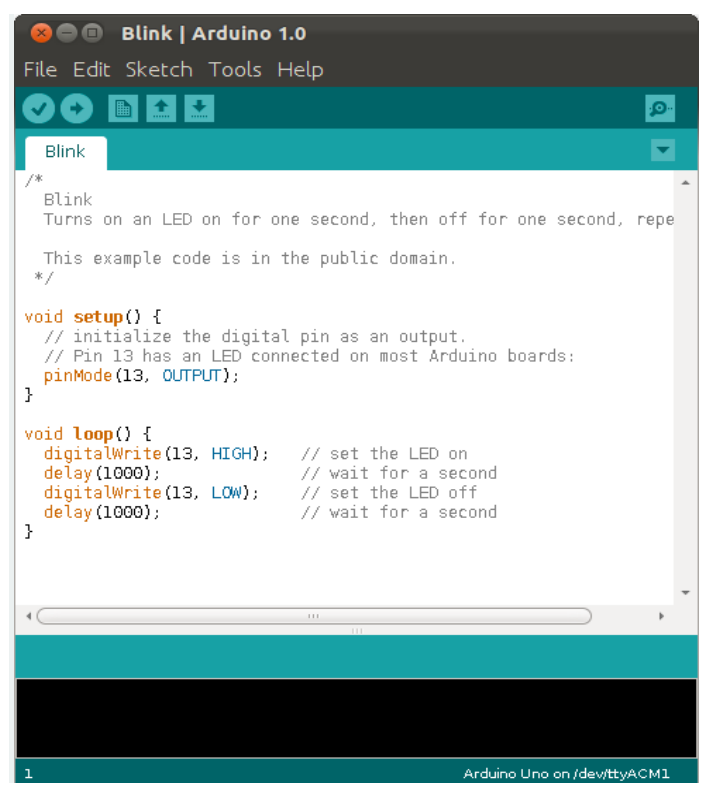

Fig4.1. Arduino IDE

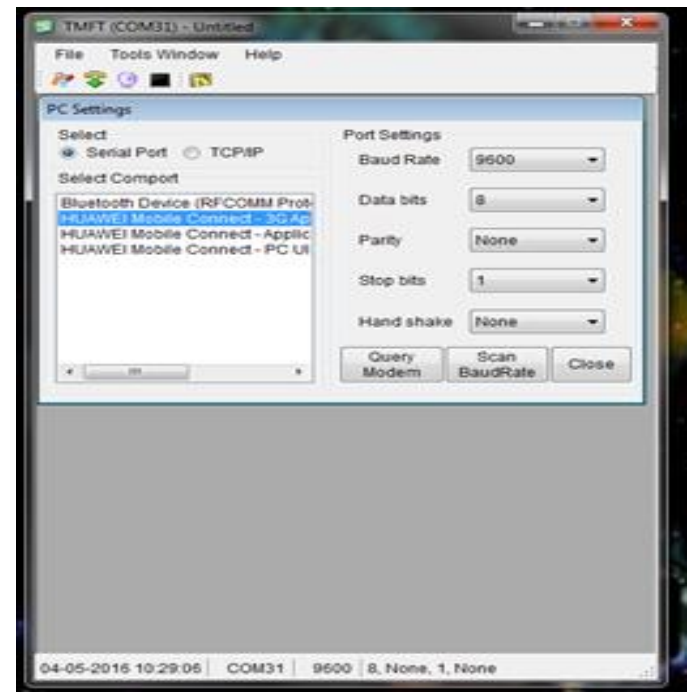

Fig4.2. TMFT terminal

TMFT is multifunctional tool for TARANG, developed by Melange systems. We will use a USB to serial connector for interacting with the SIM 800 module.

TMFT is just like the HyperTerminal in Windows. It uses an interface similar to that of "messenger" or "chat" programs, with a large window that contains all received data and an edit line for typing in strings to transmit.

The serial port as to chosen and the BAUD rate of 9600 is to be fixed. All the handshaking signals are disabled and stop bit is set to 1 .

After the setup is complete click on open terminal and wait for the response from the GSM module. When the GSM module is ready it will be indicated in the terminal by the presence of three green dots. The first command to be entered is the AT instruction. The AT instruction will gain the attention of the GSM module. The response to the AT instruction from the GSM module will be OK. Once the connection is verified, series of AT commands can be used for the exchange of information with the modem.

If there is a live incoming request or a message to the server module, an indication will be displayed in the terminal with the number of the message and SIM from which the message is originated. 


\subsection{Design Flow of the Assistive Module}

Arduino will not scan for the input on its own unlike the other microcontrollers like 8051. Every time when the status of the input is required the I/O read has to be initiated by the DigitalRead ( ) or the AnalogRead ( ) function. When we are using column scanning it is required that we read the column lines every time we output a different sequence on the row lines.

The assistive module will first initialize the audio playback board, LCD and the GSM module. Once the initialization is complete it waits for the press of the master key. Until a key is pressed the assistive module will be idle. The first key to be pressed is the master key; if it is not pressed then the other keys will not be recognized. The press of master key will announce a vocal message telling the user to enter the request choice. The master key initiates the module and the device starts scanning for the actual service request. On receiving the request it will perform the display, announce and the request transmit functions.

The lookup table is implemented using an array of pointer. Each pointer is of a type character. The advantage of using pointers is that there is no need to specify the length of the request names to be stored in the table. The access using pointer is more efficient and faster when compared to two dimensional character arrays.

The audio playback board once initiated will keep repeating the message on the selected channel. Hence it is required to deactivate the channel after the duration of the vocal message so that the audio loop is avoided.

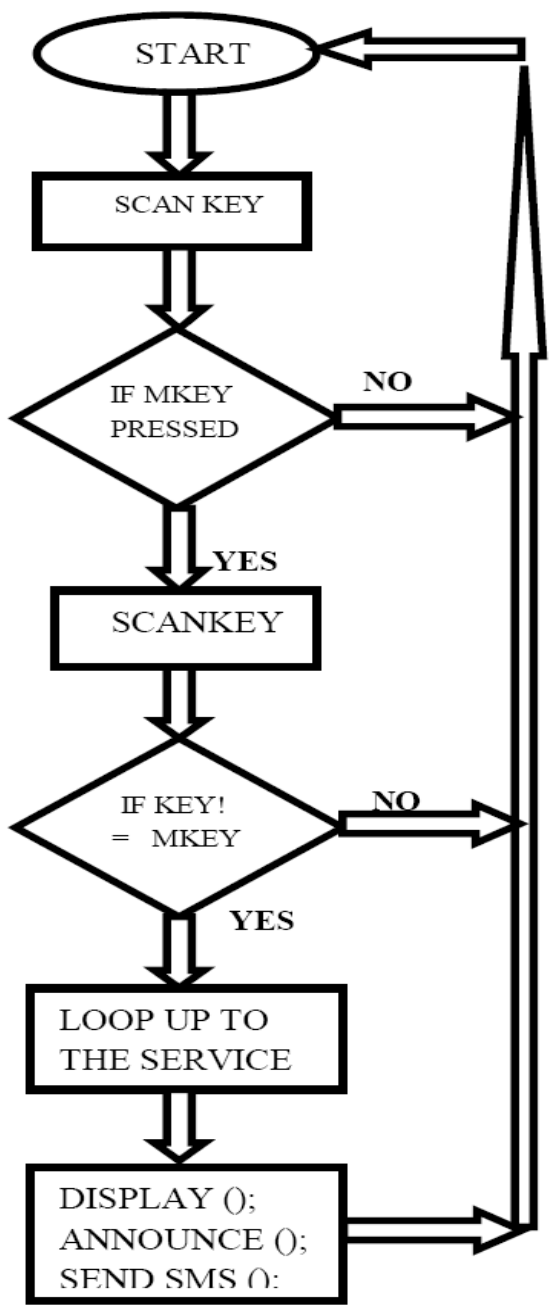

Fig4.3. Flow chart for assistive module

\section{EXPERIMENTAL RESULTS}

This section provides the results obtained from the implementation of the device. The way results are displayed on the LCD screen and the images of the messages sent using the GSM module are provided here. 

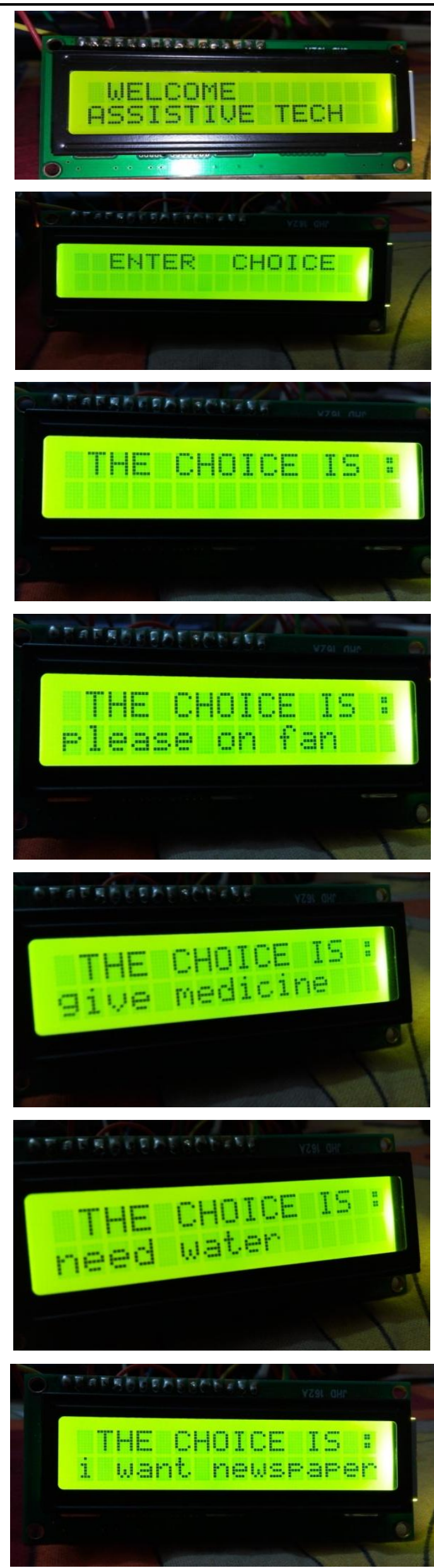

Fig5.1. Set of LCD output images 
Assistive Technology for Intellectually Disabled and Physically Challenged People

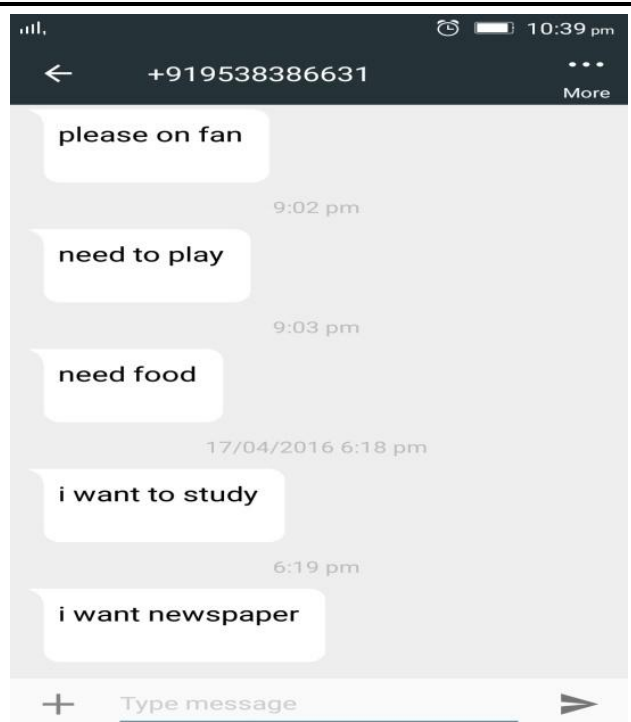

Fig5.2. Image of SMS received from GSM module
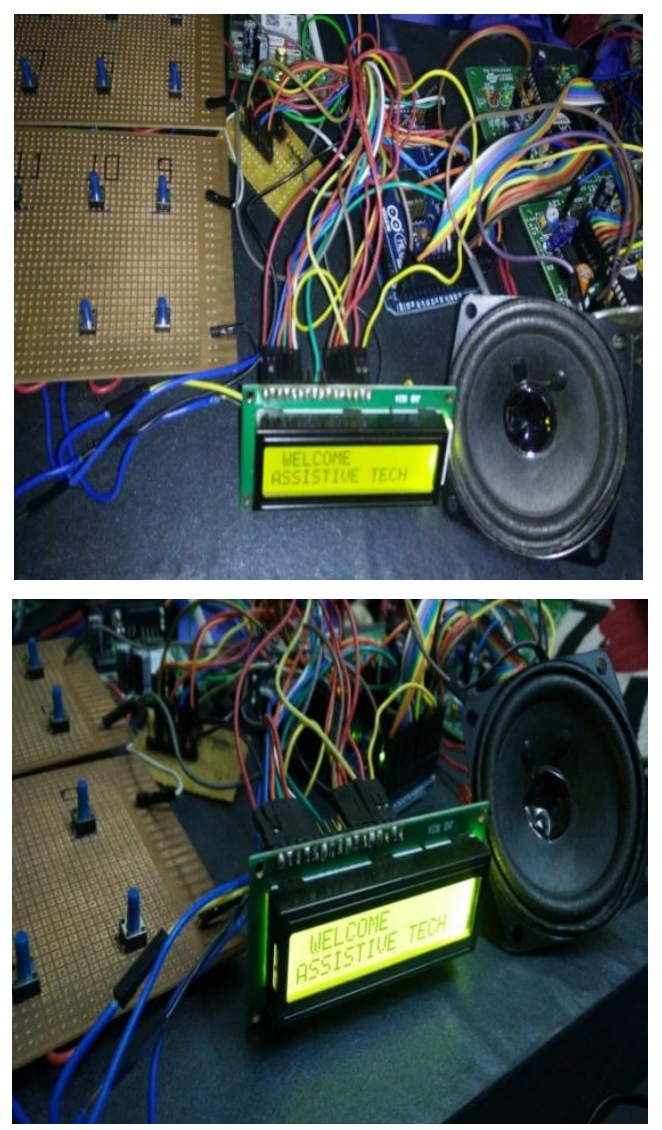

Fig5.3. Images of Integrated assistive system used for testing

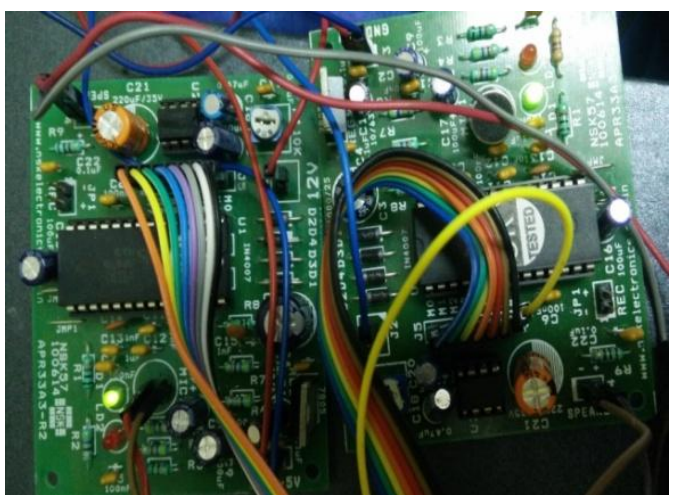

Fig5.4. Audio playback board used for vocal message 


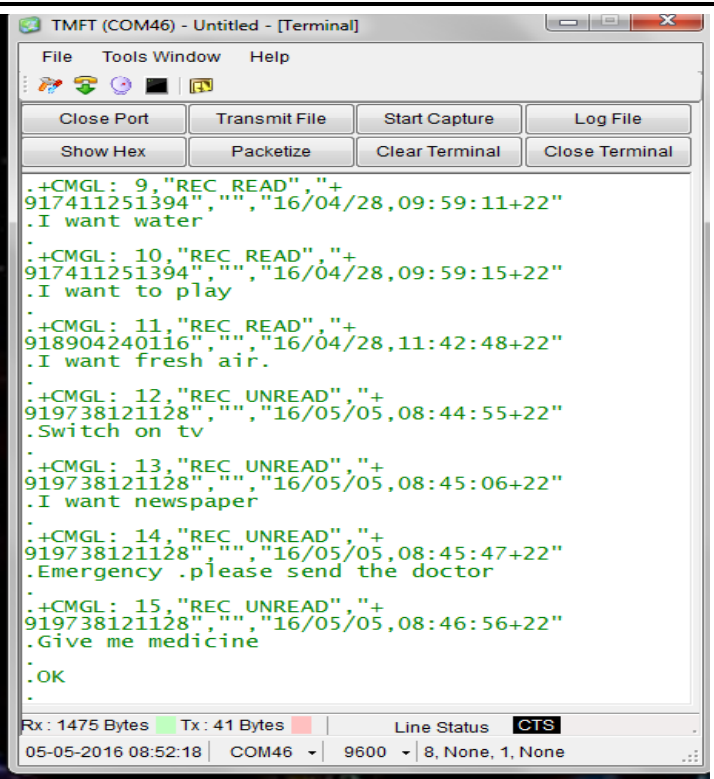

Fig5.5. TMFT terminal containing the requests received from various assistive modules

\section{Conclusion}

The assistive technology provides an easy way to train the people with intellectual disability and helps them to convey what they really need. The device also helps the assistants to clearly understand what the people with ID are trying to convey. It also alerts the assistants and provides an exact copy of the service requested in the form of a message to their cell phone. The GSM technology helps to get the service requests in the form of messages to any cell phone with slight modification in the mobile number. The device can be used in hotels for receiving the orders from the customers. The orders placed will be sent directly to the chef and the billing counter. The assistive technology can also be used in hospitals to collect the needs of the patients such as food, medicine, glucose etc...

\section{REFERENCES}

[1] GSM architecture protocol and services by Wiley (GSM communication).

[2] Data structures by padma reddy (pointer arithmetic and structures).

[3] Introduction to wireless telecommunication systems and networks by mullot (GSM architecture).

[4] Interactive numbering system for mentally challenged children-2013 IEEE (learning capabilities).

[5] Evaluation and assessment tool for mentally challenged children-2012 international conference (intellectual disability).

[6] http://www.sumeetinstruments.com/shop/index.php?route=product/product\&product_id=1013(si m800a tutorial).

[7] https://wcscnet.com/tutorials/introduction-to-rs232-serial-communication (RS232 tutorial).

[8] https://en.wikipedia.org/wiki/Arduino\#Software (arduino IDE)

[9] http://www.masters.com.pl/en,news,simcom-sim800\# (SIM 800) 


\section{AUTHORS' BIOGRAPHY}

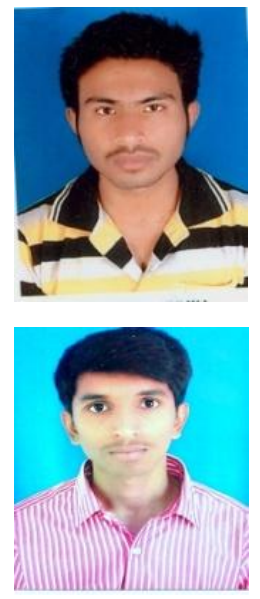

Mr.Darshan.B, Pursuing BE (E\&C) in BNM institute of technology, Banglore, Karnataka, India. This paper is based on the project carried out in the final year of BE.

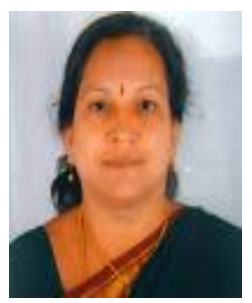

Dr. P. A. Vijaya, Did her B.E. from MCE, Hassan and M.E. and Ph.D. from IISc, Bengaluru.She worked in MCE, Hassan, Karnataka, for about 27 years and 3 years in BNMIT, Banglore, Karnataka. Presently, she is a Professor and HOD in the Dept. of E\&C Engg, BNMIT, Banglore, Karnataka, India, from 2016. Three students have obtained Ph.D degree under her guidance and four more are doing Ph.D. Her research interests are in the areas of Pattern Recognition, Image Processing, VLSI Design, Embedded Systems and RTOS. 